\title{
Modelling Considerations for Coupled Lines in CMOS Back- End-Of-Line at mm-Wave Frequencies
}

This paper was downloaded from TechRxiv (https://www.techrxiv.org).

\section{LICENSE}

CC BY-NC-SA 4.0

SUBMISSION DATE / POSTED DATE

$12-04-2021 / 25-01-2022$

CITATION

Venter, Johannes; Stander, Tinus (2021): Modelling Considerations for Coupled Lines in CMOS Back-End-OfLine at mm-Wave Frequencies. TechRxiv. Preprint. https://doi.org/10.36227/techrxiv.14401661.v2

$\mathrm{DOI}$

10.36227/techrxiv.14401661.v2 


\title{
Modelling Considerations for Coupled Lines in CMOS Back-End-Of-Line at mm-Wave Frequencies
}

\author{
Johannes J. P. Venter and Tinus Stander
}

\begin{abstract}
We investigate the effect of passivation contouring, surface roughness, and sidewall tapering on the FEM modelling accuracy of mm-wave couplers in CMOS BEOL. Of three effects, sidewall tapering leads to the most significant improvement $(0.37$ dB) in predicting peak coupling magnitude at V-band. Ultimately, it is found that none of these measures substantially improve on modelling accuracy.
\end{abstract}

Keywords-CMOS technology, directional couplers, finite element analysis, numerical simulation.

\section{INTRODUCTION}

mm-Wave integrated circuit (IC) transceivers have become commonplace in communications, automotive RADAR and a variety of other applications, due in part to the availability of suitable low-cost CMOS and SiGe BiCMOS processes. These require accurate performance estimation of integrated mmwave passives on-chip, such as coupled line filters [1] and couplers [2], typically aided by full-wave FEM simulation [3], [4]. One non-ideality often overlooked in modelling distributed passives in CMOS is the sidewall taper (i.e. vertical line edges not perpendicular to the wafer) on metal lines [5]. This has been shown to affect the static capacitances of side-by-side lines onchip [6] and reflecting structures for CMOS light sources [7]. In addition, sidewall taper has been known to affect distributed coupling and line impedance on RF PCB [8]. Another nonideality that is often neglected in simulating on-chip passives is surface roughness, even though surface roughness of back-endof-line (BEOL) can be in the order of $5 \%$ of the metal thickness [9]. Finally, there is the contour of the passivation layer covering the top metal [7], which is often neglected in a $2.5 \mathrm{D}$ stack-up modelling approach even if a 3D FEM mesh is generated.

This work presents the first comparative simulation study with experimental verification on the effect of passivation contouring, sidewall tapers, and surface roughness on the broadband, mm-wave coupling of coplanar lines (i.e. both conductors on the top metal layer) in a CMOS back-end-of-line (BEOL) stack-up.

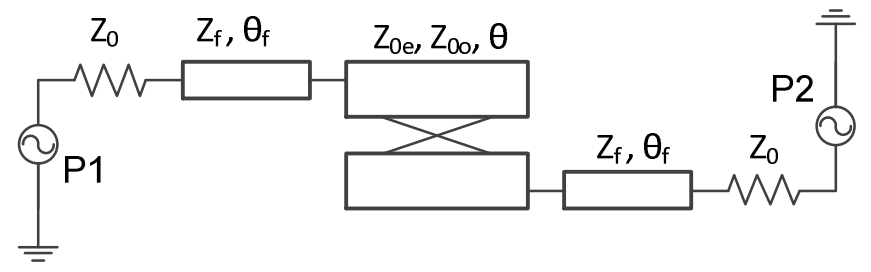

Fig. 1. Circuit under test.

\section{CIRCUIT UNDER TEST}

The test circuit is shown in Fig. 1, and consists of two coupled lines terminated by open-circuits at opposite ends, with short feed lines. It is one of the 10 canonical forms of the general four-port directional coupler [10], [11], and is selected not only for its specific applicability to coupled line coupler and filter synthesis, but also because it can easily be fabricated onchip and evaluated with a two-port VNA.

The directional coupling $C$ can be calculated from an Sparameter measurement by first considering [11]

$$
Z_{0 e}=Z_{0} \sqrt{\frac{1+C}{1-C}}, Z_{0 o}=Z_{0} \sqrt{\frac{1-C}{1+C}}
$$

which can be substituted into expressions for Z-parameters of the network in Fig. 1 (assuming the feed lines are deembedded) as [12]

$$
\begin{aligned}
& Z_{11}=Z_{22}=-\frac{j}{2}\left(Z_{0 e}+Z_{0 o}\right) \cot (\theta) \\
& Z_{21}=Z_{12}=-\frac{j}{2}\left(Z_{0 e}-Z_{0 o}\right) \csc (\theta)
\end{aligned}
$$

after which S-parameters can be derived from standard twoport matrix transformation. At the frequency where $\theta=\pi / 2$, it can be shown that

$$
\begin{aligned}
& \left.S_{11}\right|_{\theta=\pi / 2}=\left.S_{22}\right|_{\theta=\pi / 2}=2 C^{2}-1 \\
& \left.S_{21}\right|_{\theta=\pi / 2}=\left.S_{12}\right|_{\theta=\pi / 2}=\frac{2 j C\left(C^{2}-1\right)}{\sqrt{1-C} \sqrt{1+C}}
\end{aligned}
$$

Similarly, by relating the coupled line admittance inverter value $J$ to $Z_{0 e}$ and $Z_{0 o}$ as [12]

$$
\begin{aligned}
& Z_{0 e}=Z_{0}\left(1+J Z_{0}+\left(J Z_{0}\right)^{2}\right) \\
& Z_{0 o}=Z_{0}\left(1-J Z_{0}+\left(J Z_{0}\right)^{2}\right)
\end{aligned}
$$

and again substituting into (2) and (3), expressions for the Sparameters at the coupling centre frequency may be obtained as 


$$
\begin{aligned}
& \left.S_{11}\right|_{\theta=\pi / 2}=\left.S_{22}\right|_{\theta=\pi / 2}=\frac{J^{2} z_{0}^{2}-1}{J^{2} Z_{0}^{2}+1} \\
& \left.S_{21}\right|_{\theta=\pi / 2}=\left.S_{12}\right|_{\theta=\pi / 2}=\frac{-2 j J Z_{0}}{J^{2} Z_{0}^{2}+1}
\end{aligned}
$$

S-parameter measurements on the network in Fig. 1 could, therefore, be used to experimentally investigate process variation on either $C$ (for directional couplers) or $J$ (for coupled line filters), and is therefore used as a test vehicle to investigate coupling here.

\section{PROTOTYPE DEVICES}

To evaluate the simulation results, three geometries under test were fabricated (as shown in Fig. 2) and analysed on an Anritsu ME7828A VNA. In all cases, the $150 \mu \mathrm{m}$ pitch GSG pads were de-embedded from measurement with on-chip TRL standards, to align the measurement calibration plane with the simulated ports. The reference planes are placed $68 \mu \mathrm{m}$ from the pad edges, as indicated in red on Fig. 2(a) for the top coupler.

The coupler under investigation (Fig. 3) is implemented on the ams C35 process (4 metal layer BEOL) [13]. It is implemented as microstrip between the M4 top metal layer and a finite M1 ground plane, coupling two lines of width $w=7.88$ $\mu \mathrm{m}$ and length $l=522.6 \mu \mathrm{m}$ over a separation $d=1,4$, and 7 $\mu \mathrm{m}$ in three experiments. The coupler is fed at an angle $\theta_{f}=$ $135^{\circ}$ with two microstrip lines of $w_{f}=7.88 \mu \mathrm{m}$ and $l_{f}=202 \mu \mathrm{m}$.

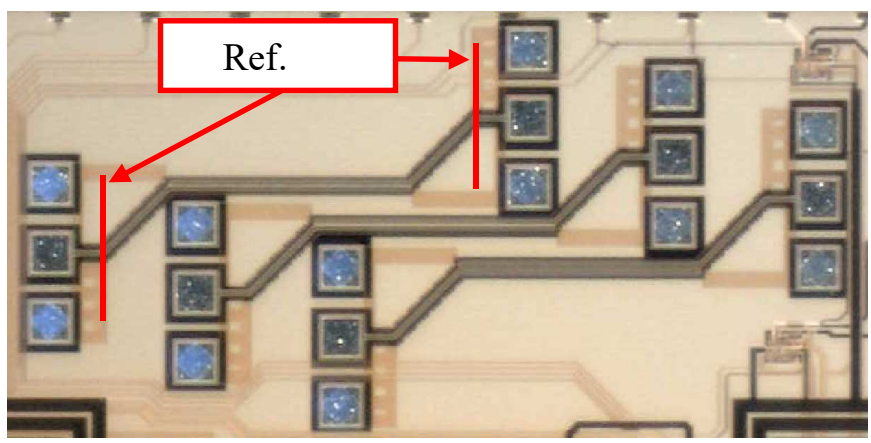

(a)

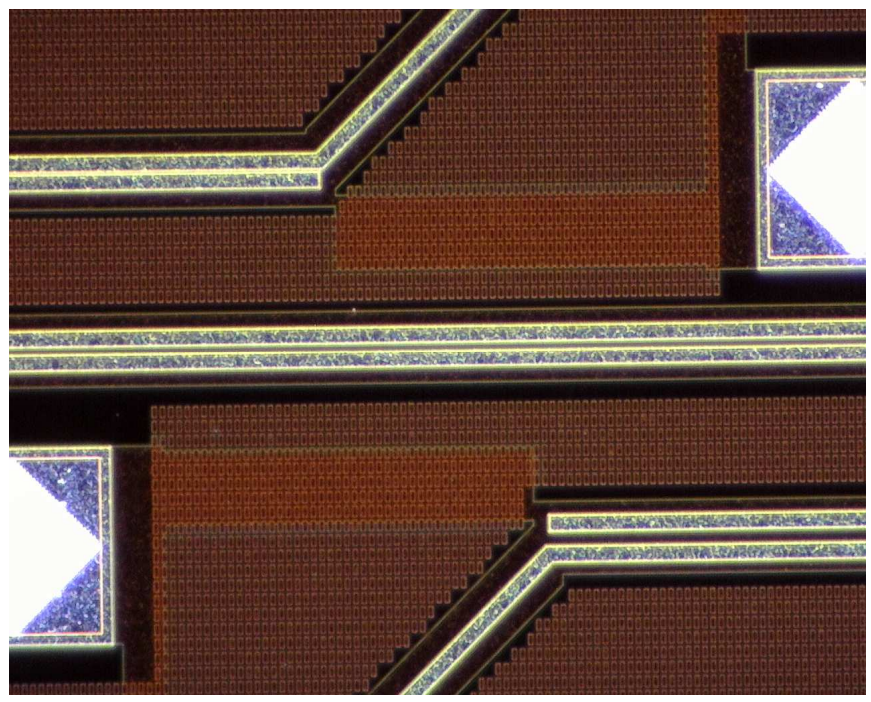

(b)

Fig. 2. Prototyped couplers. (a) Full layout showing reference planes, (b) Detail view.

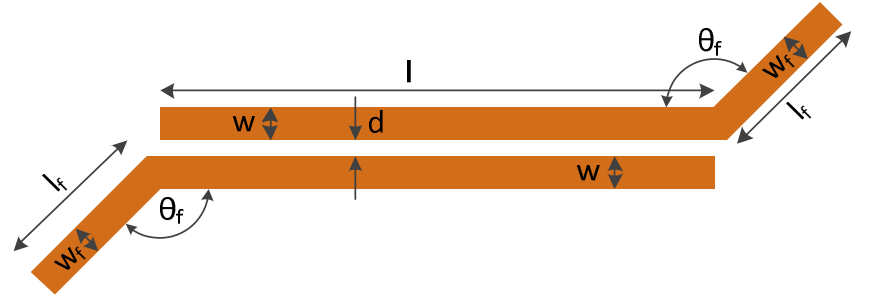

Fig. 3. Geometry of coupler.

\section{SIMULATION STUDY}

With the prototypes and measurement results in place, different approaches are applied to modelling the couplers in a commercial FEM solver [4] to evaluate their accuracy in predicting the measured responses. All simulations were done using ANSYS Electronics Desktop. All couplers were simulated in isolation, to limit the simulation mesh size. As the spacing between each coupler and the GSG pads of an adjacent coupler is more than 5 times the dielectric thickness between M4 and M1, the influence of stray coupling is considered negligible.

\section{A. Passivation layer modelling}

The 2.5D layered passivation models, as shown in Fig. 4(a), are constructed as HFSS 3D layout designs and simulated using the HFSS 3D FEM solver with the gap port inductance deembedded. In addition, two models are constructed using the 3D modelling interface; one using the same flat passivation (Fig. 4(a)), and the other using a contoured passivation (Fig. 4(b)). Both 3D models are excited using wave ports. Surface roughness and sidewall taper are neglected, initially.

Fig. 5 shows the simulation results $\left(\left|\mathrm{S}_{21}\right|\right)$ obtained for the different passivation modelling options compared with the measured results for the $d=1,4$, and $7 \mu \mathrm{m}$ experiments, indicating no significant improvement in modelling accuracy.

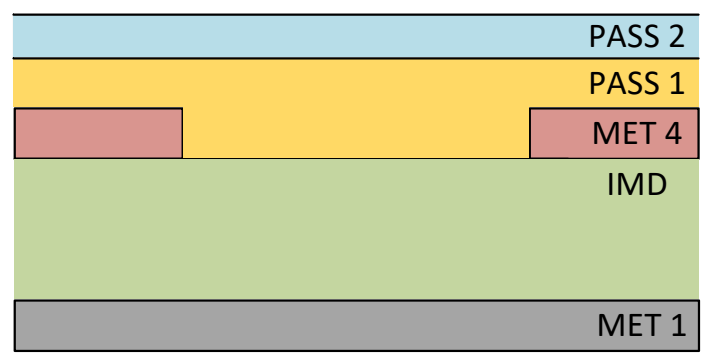

(a)

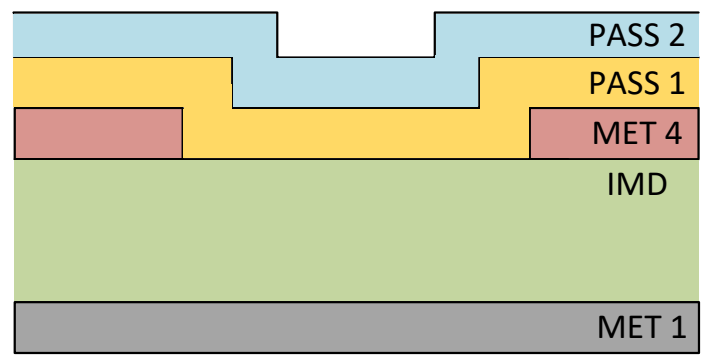

(b)

Fig. 4. Cross-sectional views of different passivation stack-up options. (a) Flat passivation, (b) Contoured passivation 


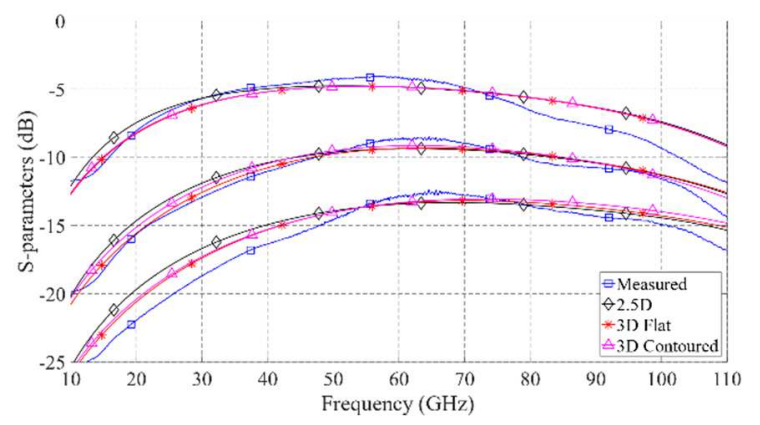

Fig. 5. Comparison of simulation results $\left(\left|\mathrm{S}_{21}\right|\right)$ of stack-up options for $\mathrm{d}=1$, $4,7 \mu \mathrm{m}$.

\section{B. Surface roughness effects}

The RMS surface roughness values for M4 analyzed here are $46.25 \mathrm{~nm}$ and $92.5 \mathrm{~nm} ; 5 \%$ and $10 \%$ of the metal thickness [9]. The surface roughness is modelled in HFSS using the Huray "snowball" model [14] with $A_{\text {matte }} / A_{\text {flat }}=1$ and 14 equalradius spheres of nodule radii of $9.5787 \mathrm{~nm}$ and $19.1574 \mathrm{~nm}$ for the two cases, with the Hall-Huray Surface Ratio at 4.8869 .

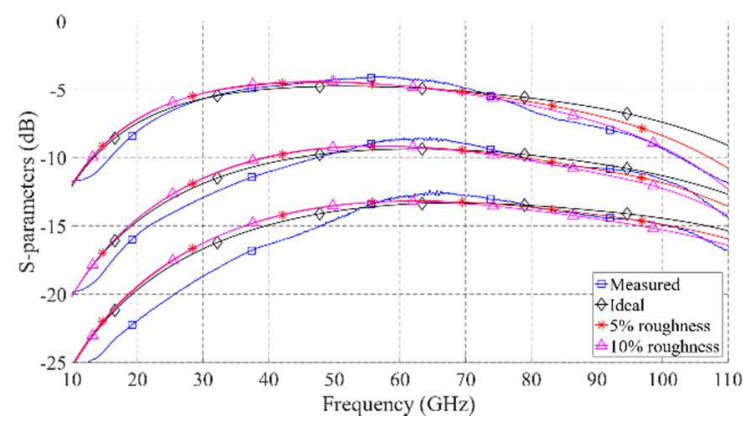

(a)

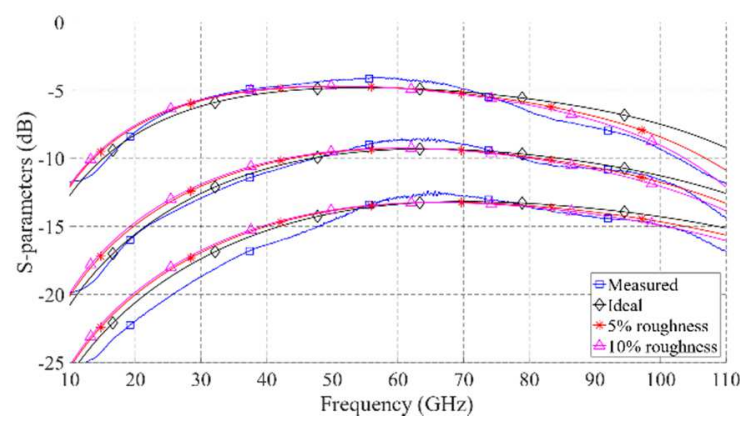

(b)

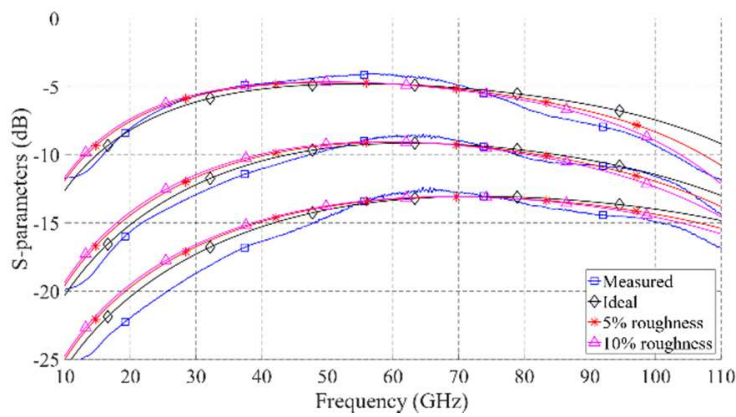

(c)

Fig. 6. Surface roughness effect on simulation results (|S21|) of couplers for $\mathrm{d}=1,4,7 \mu \mathrm{m}$. (a) 2.5D flat passivation, (b) 3D flat passivation, (c) 3D contoured passivation
TABLE I

RMS ERROR OF $\left|\mathrm{S}_{21}\right|$ FOR VARIOUS SURFACE ROUGHNESS VALUES

\begin{tabular}{lccc}
\hline \hline Passivation & \multicolumn{3}{c}{ Surface roughness } \\
& Ideal & $5 \%$ & $10 \%$ \\
\hline 2.5D Flat & 0.0330 & 0.0266 & 0.0246 \\
3D Flat & 0.0309 & 0.0226 & 0.0209 \\
3D Contour & 0.0315 & 0.0246 & 0.0234 \\
\hline \hline
\end{tabular}

The RMS error over the band of interest, shown in Table 1, as well as the comparison in Fig. 6 would indicate that, while lower error is achieved when accounting for surface roughness, the improvement is only marginal.

\section{Sidewall taper effects}

The sidewall taper variations (indicated by $\alpha$ in Fig. 7(a)) are selected from measurements and estimates in literature [7]-[9] as $75^{\circ}, 82^{\circ}, 104^{\circ}$, and $121^{\circ}$, with $90^{\circ}$ retained as nominal reference. The drawn width ( $X$ in Fig. 7(a) or $W$ in Fig. 3) of the coupled lines were kept constant throughout the analysis. A mesh detail view of the sidewall taper modelled in HFSS 3D layout is shown in Fig. 7(b).

The maximum coupling value varies by $0.8 \mathrm{~dB}$, on average, across the different passivation options, as $\alpha$ varies from $75^{\circ}$ to $121^{\circ}$ (shown in Fig. 9), with $104^{\circ}$ producing results that best agree with measurement. Fig. 8 shows the simulation results, with Table 2 summarizing the RMS error for $\left|S_{21}\right|$ across a frequency band of $40 \mathrm{GHz}-80 \mathrm{GHz}$. From these results, it is evident that an edge taper angle of $104^{\circ}$ yields the most accurate results when using the 3D passivation layer modelling approaches, though only by a small margin.

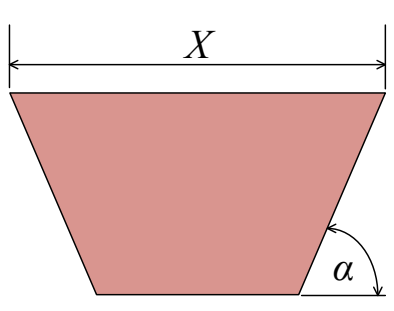

(a)

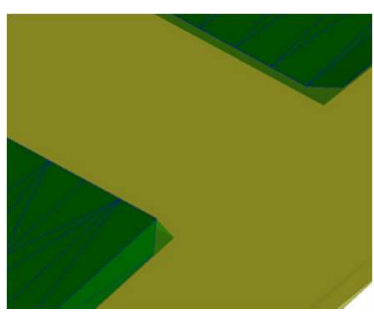

(b)
Fig. 7. Sidewall taper modelling. (a) Geometry, (b) mesh detail view.

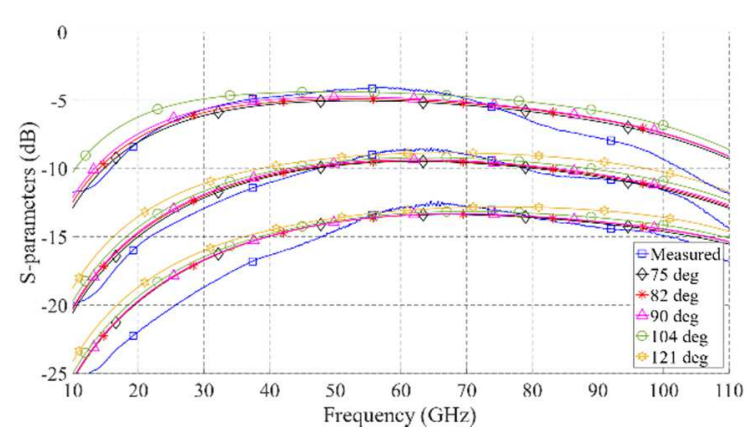

(a) 


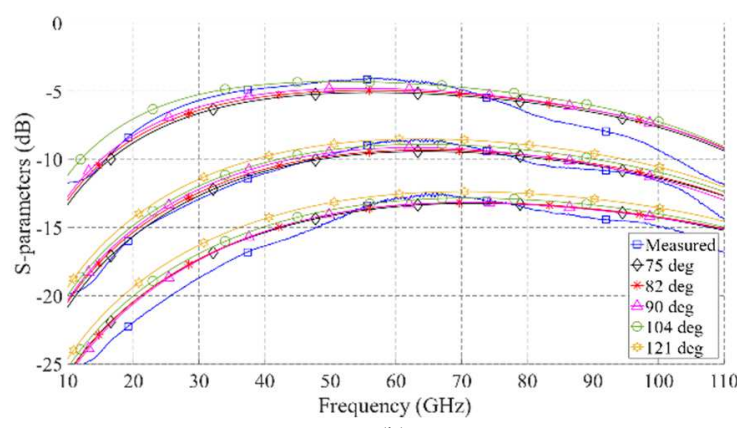

(b)

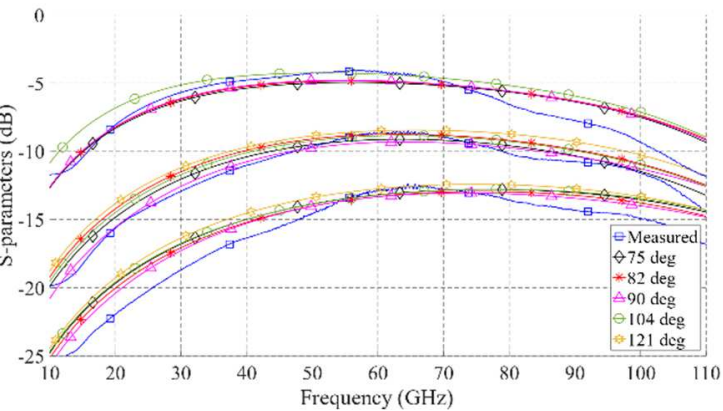

(c)

Fig. 8. Sidewall taper effect on simulation results $\left(\left|\mathrm{S}_{21}\right|\right)$ of couplers for $\mathrm{d}=1,4,7 \mu \mathrm{m}$. (a) 2.5D flat passivation, (b) 3D flat passivation, (c) $3 \mathrm{D}$ contoured passivation

TABLE II

RMS ERROR OF $\left|\mathrm{S}_{21}\right|$ FOR VARIOUS EDGE TAPER ANGLES

\begin{tabular}{lccccc}
\hline \hline Passivation & \multicolumn{5}{c}{ Taper angles $(\alpha$ in Fig. 7.) } \\
& $75^{\circ}$ & $82^{\circ}$ & $90^{\circ}$ & $104^{\circ}$ & $121^{\circ}$ \\
\hline 2.5D Flat & 0.0307 & 0.0274 & 0.0241 & 0.0223 & 0.0198 \\
3D Flat & 0.0322 & 0.0276 & 0.0232 & 0.0193 & 0.0258 \\
3D Contour & 0.0271 & 0.0258 & 0.0236 & 0.0207 & 0.0263 \\
\hline \hline
\end{tabular}

\section{DISCUSSION}

From these results, it is evident that the $3 \mathrm{D}$ contoured passivation layer modelling approach yields fractionally better modelling accuracy, improving the peak coupling prediction error from $0.71 \mathrm{~dB}$ to $0.56 \mathrm{~dB}$ when compared to the simplest 2.5D uniform layer passivation approach.

Surface roughness is found to increase losses at higher frequencies, but also increases the maximum coupling value and lowers the frequency of peak coupling. The most accurate value $(46.25 \mathrm{~nm}$ ) yields $0.514 \mathrm{~dB}$ and $2.87 \mathrm{GHz}$ modelling error in peak coupling and centre frequency, compared to 0.56 $\mathrm{dB}$ and $2.79 \mathrm{GHz}$ error when neglecting surface roughness.

Sidewall taper is shown to have a marginally stronger effect on predicting the peak coupling value, with an average of 0.8 $\mathrm{dB}$ of change between different taper values. The most accurate results were obtained using $104^{\circ}$ (from [7]), producing $0.19 \mathrm{~dB}$ error compared to the $0.56 \mathrm{~dB}$ using perfect $90^{\circ}$ edges.

The simulation times for $2.5 \mathrm{D}, 3 \mathrm{D}$ flat passivation, and $3 \mathrm{D}$ contoured passivation were $10 \mathrm{mins}, 7 \mathrm{mins}$, and $5 \mathrm{mins}$, respectively; therefore, apart from the minor improvement of using a 3D model, there is also an improvement in simulation time when using the $3 \mathrm{D}$ modelling approaches.

\section{CONCLUSION}

Different approaches to 3D FEM modelling of passivation, RMS surface roughness, and sidewall edge taper in mm-wave on-chip couplers, have been evaluated. It is found that neither the application of a 3D contoured passivation layer, nor surface roughness, substantially improve the modelling accuracy, while accurate sidewall tapering only marginally improved modelling accuracy.

\section{ACKNOWLEDGMENT}

This work was sponsored by the National Research Foundation of South Africa (NRF) under grants 92526 and 93921, as well as the South African Radio Astronomy Observatory (SARAO) and the Eskom Tertiary Education Support Programme (TESP).

\section{REFERENCES}

[1] S. Sun, J. Shi, L. Zhu, S. C. Rustagi, and K. Mouthaan, "MillimeterWave Bandpass Filters by Standard 0.18- $\mathrm{m}$ CMOS Technology," IEEE Electron Device Lett., vol. 28, no. 3, pp. 220-222, Mar. 2007.

[2] J. Lugo-Alvarez, A. Bautista, F. Podevin, and P. Ferrari, "Highdirectivity compact slow-wave CoPlanar waveguide couplers for millimeter-wave applications," in 44th European Microwave Conference, 2014, pp. 1072-1075.

[3] F. Sagouo Minko and T. Stander, "EM Modelling Considerations for mm-Wave On-Chip Antennas," IOP Conf. Ser. Mater. Sci. Eng., vol. 766, p. 012001, Mar. 2020.

[4] F. Sagouo Minko and T. Stander, "A comparison of three-dimensional electromagnetic and RC parasitic extraction analysis of mm-wave onchip passives in SiGe BiCMOS low-noise amplifiers," Int. J. RF Microw. Comput. Eng., vol. 30, no. 2, 2020.

[5] K. Powell, S. Burgess, T. Wilby, R. Hyndman, and J. Callahan, "3D IC Process integration challenges and solutions," in 2008 International Interconnect Technology Conference, 2008, pp. 40-42.

[6] R. T. Elsayed, A. R. Farhang, and J. C. Yip, "Interconnect Characterization: Accuracy, Methodology, and Practical Considerations," IEEE Trans. Semicond. Manuf., vol. 21, no. 3, pp. 435443, Aug. 2008.

[7] A. W. Bogalecki, M. du Plessis, P. J. Venter, I. J. Nell, and M. E. Goosen, "Integrated optical light directing structures in CMOS to improve light extraction efficiency," in 2010 International Conference on Microelectronics, 2010, pp. 168-171.

[8] A. Renbi, A. Risseh, R. Qvarnström, and J. Delsing, "Impact of etch factor on characteristic impedance, crosstalk and board density," in 45th International Symposium on Microelectronics, 2012, vol. 2012, no. 1, pp. 312-317.

[9] J. A. Davis, R. Venkatesan, A. Kaloyeros, M. Beylansky, S. J. Souri, K. Banerjee, K. C. Saraswat, A. Rahman, R. Reif, and J. D. Meindl, "Interconnect limits on gigascale integration (GSI) in the 21 st century," Proc. IEEE, vol. 89, no. 3, pp. 305-324, Mar. 2001.

[10] G. I. Zysman and A. K. Johnson, "Coupled Transmission Line Networks in an Inhomogeneous Dielectric Medium," IEEE Trans. Microw. Theory Tech., vol. 17, no. 10, pp. 753-759, Oct. 1969.

[11] D. M. Pozar, Microwave engineering, 4th ed. Hoboken, NJ: Wiley, 2012.

[12] J.-S. Hong and M. J. Lancaster, Microstrip Filters for RF / Microwave Applications. John Wiley \& Sons, Ltd, 2004.

[13] J. J. P. Venter, A.-L. Franc, T. Stander, and P. Ferrari, "Transmission Lines Characteristic Impedance vs. Q-factor in CMOS Technology," Int. J. Microw. Wirel. Technol., vol. (accepted), 2021.

[14] X. Ye, "From surface roughness modeling to surface roughness engineering signal integrity and power integrity," IEEE Electromagn. Compat. Mag., vol. 9, no. 2, pp. 60-61, 2020. 